\title{
Der Einfluß von thermozyklischer Beanspruchung auf den Eigenspannungs- zustand in elektronenstrahlgedampften Wärmedämmschichten aus Zirkonoxid
}

\author{
H. Richter, W. Wei und S. Schneiderbanger
}

\begin{abstract}
Flachproben und Turbinenschaufeln für Flugtriebwerke wurden mit elektronenstrahlgedampften Wärmedämmschichten aus tejlstabilisiertem Zirkonoxid beschichtet. Der Eigenspannungszustand vor und nach Thermowechselbelastung wurde röntgenografisch bestimmt. An allen Flachproben und Schaufeln wurden Druckeigenspannungen gemessen. Die Eigenspannungen waren in Probenlängsrichtung signifikant höher als in der Querrichtung. Eine Änderung des Eigenspannungszustandes als Folge der thermozyklischen Beanspruchung wurde nicht beobachtet. Die Proben wiesen stark unterschiedliche Texturzustände auf, welche die Auswertung der Eigenspannungsmessung und möglicherweise auch das Schichtverhalten gegenüber thermozyklischer Beanspruchung beeinflußten.
\end{abstract}

\section{Influence of Thermal Cycling on Residual Stresses in Zirconia Thermal Barrier Coatings Produced by Electron Beam - Physical Vapor Deposition}

Flat specimens and aircraft turbine engine blades were coated with partially stabilized zirconia by electron beam - physical vapor deposition. Residual stresses of these thermal barrier coatings were measured before and after thermal cycling by $x$-ray stress analysis. All flat specimens and blades showed compressive residual stresses. Stress values in the longitudinal direction were significantly greater than those measured in the transverse direction. No changes of stress states and line widths were observed after thermal cycling. The specimens showed considerable differences in texture which made evaluation of the stress measurements difficult and which may have influenced degradation behaviour during thermal cycling.

\section{Einleitung}

Wärmedämmschichten aus teilstabilisiertem Zirkonoxid werden im Flugtriebwerksbau eingesetzt, um die Lebensdauer von gekühlten Komponenten zu erhöhen und/oder um den thermischen Wirkungsgrad durch höhere Betriebstemperaturen zu steigern. Bisherige Wärmedämmschichten wurden durch das Plasmaspritzverfahren hergestellt. Thermozyklische Versuche mit elektronenstrahlgedampften Schichten haben ergeben, daß sich mit diesen Schichten wesentlich längere Standzeiten ohne erkennbare Schäden erzielen lassen als mit plasmagespritzten Schichten [1]. Dies wird vor allem auf den unterschiedlichen Gefügeaufbau der beiden Schichtarten zurückgeführt.

In einer vorangegangenen Arbeit wurde gezeigt, daß bei plasmagespritzten $\mathrm{ZrO}_{2}$-Wärmedämmschichten auch der Eigenspannungszustand der Schichten deren Lebensdauer stark beeinflußt [2]. Röntgenografische Untersuchungen ergaben, daß das Versagen der plasmagespritzten Schichten bei thermozyklischer Beanspruchung mit der Zunahme von Druckeigenspannungen in den Schichten einhergeht und daß durch gezielte Beeinflussung der Eigenspannungen im Ausgangszustand die Lebensdauer der Schichten gesteigert werden kann. Ziel der jetzigen Arbeit war, zu klären, ob das unter thermozyklischer Beanspruchung letzten Endes auch bei elektronenstrahlgedampften Schichten auftretende Schichtversagen durch Abplatzen ebenfalls mit Änderungen des Eigenspannungszustandes einhergeht.

\section{Probenherstellung}

Röntgenografische Eigenspannungsmessungen wurden an Flachproben und an typischen Turbinenlaufschaufeln durchgeführt, $A b b .1$. In beiden Fällen war der Grundwerkstoff die Ni-Basislegierung IN100 $(15 \mathrm{Co}, 9,5 \mathrm{Cr}$, 5,5 Al, $5 \mathrm{Ti}, 3 \mathrm{Mo}$, andere Zusatzelemente, Rest Ni). Das Wärmedämmschichtsystem bestand aus einer NiCoCrAlYHaftschicht und einer $\mathrm{ZrO}_{2}-7 \mathrm{Y}_{2} \mathrm{O}_{3}$ teilstabilisierten Wärmedämmschicht. Die Schichten wurden von der Firma Chromalloy Research and Technology, USA mit dem Elektronenstrahldampfverfahren hergestellt. Die Dicke der Haftschicht betrug etwa $100 \mu \mathrm{m}$ und die der Wärmedämmschicht etwa $250 \mu \mathrm{m}$.

Bei dem Elektronenstrahldampfverfahren (EB-PVD) wird der zu beschichtende Gegenstand über einem (oder ggf. mehreren) Target aus dem Schichtmaterial montiert. Durch einen Elektronenstrahl wird die Oberfläche des Targets geschmolzen und verdampft. Der Dampf schlägt sich auf dem Gegenstand nieder. Durch u.a. die Strahlleistung, den Abstand des Gegenstands zur Schmelze, die Beschichtungszeit und die atmosphärischen Bedingungen in der Beschichtungskammer kann die Qualität der Schicht kontrolliert werden. Die Flachproben wurden einseitig beschichtet, wogegen die Schaufeln während der Beschichtung parallel zur Schaufellängsachse und zur Schmelzoberfläche rotiert wurden, so daß eine gleichmäßige Rundumbeschichtung des Schaufelblatts zustande kam. 


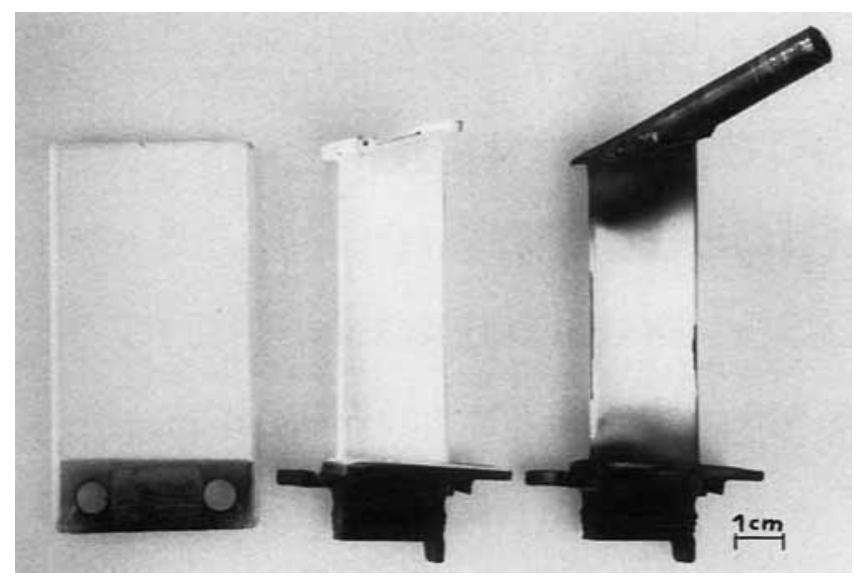

Abb. 1. Elektronenstrahlgedampfte Flachprobe (links) und Turbinenschaufel (mitte) im Ausgangszustand. Rechts: Turbinenschaufel nach thermozyklischer Beanspruchung; Schichtablösungen an Ein- und Austrittskante

Fig. 1. Flat specimen (left) and turbine engine blade (middle) with electron beam vapor deposited zirconia coating in original state. The right blade shows delamination at lcading and trailing edge after thermal cycling

Die Mikrostrukturen von EB-PVD-Schichten unterscheiden sich von herkömmlichen plasmagespritzten Schichten dadurch, daß sie aus Stengeln bestehen, die senkrecht (d.h. in Dampfrichtung) zur Oberfläche stehen (vgl. $A b b .2$ und 3). Es wird vermutet, daß der Grund für die besseren Eigenschaften der EB-PVD Keramikschichten vor allem in der höheren "Nachgiebigkeit“ der stengeligen Struktur parallel zur Oberfläche liegt.

\section{Versuchsverfahren}

\subsection{Thermoermüdung}

Die Flachproben und Schaufeln wurden in einem Heißgasprüfstand thermozyklisch belastet. Eine detaillierte Beschreibung des Prüfstands sowie die Prüfbedingungen sind [2] zu entnehmen. Kurz zusammengefaßt, wurden die Proben abwechselnd $30 \mathrm{~s}$ im Heißgas gehalten (Heißgastemperatur $=1485^{\circ} \mathrm{C}$ ) und $30 \mathrm{~s}$ mit Preßluft abgekühlt (untere Temperatur $=$ etwa $150^{\circ} \mathrm{C}$ ). Während die Flachproben ohne rückseitige Kühlung geprüft wurden, wurden die Schaufeln durch deren vorhandene Kühiluftbohrungen so gekühlt, daß die Haftschicht etwa $1120^{\circ} \mathrm{C}$ maximale Temperatur erreichte. Die Schaufeln wurden bis zu ersten Abplatzungen an der Eintrittskante geprüft. Derartige Abplatzungen gelten als Versagenskriterium für die Wärmedämmschicht.

\subsection{Röntgendiffraktometrie und röntgenografische Spannungsmessung}

Röntgendiffraktometrische Messungen wurden mit einem Siemens Omega-Goniometer D500, Kupfer Kalpha-Strahlung und einem ortsempfindlichen Detektor mit Metalldraht vorgenommen. Die Auswertung erfolgte mit den Programmen Diffrac 11 bzw. Stress 11. Die Lage und Größe der Meßstellen an den Flachproben und

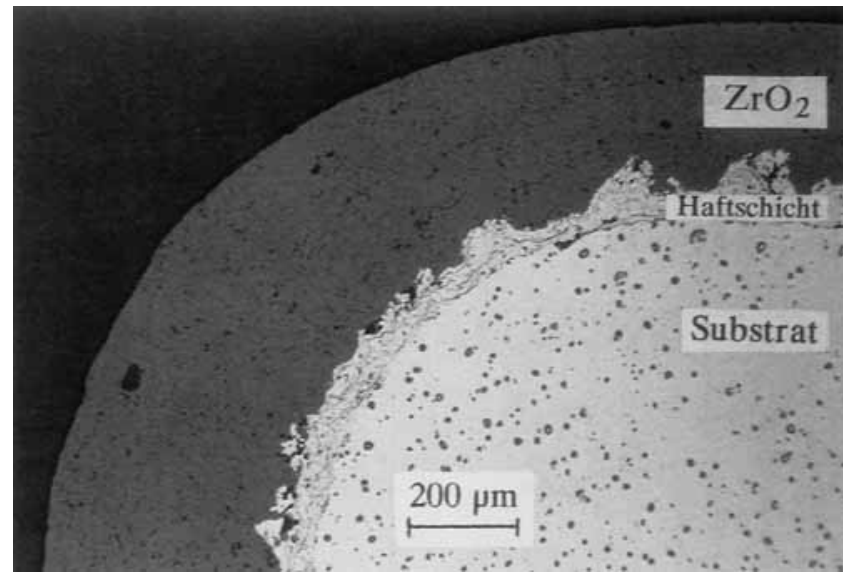

Abb. 2. Schliffbild einer plasmagespritzten Zirkonoxidschicht mit poröser Mikrostruktur

Fig. 2. Metallographic section of a plasma sprayed zirconia coating with porous microstructure

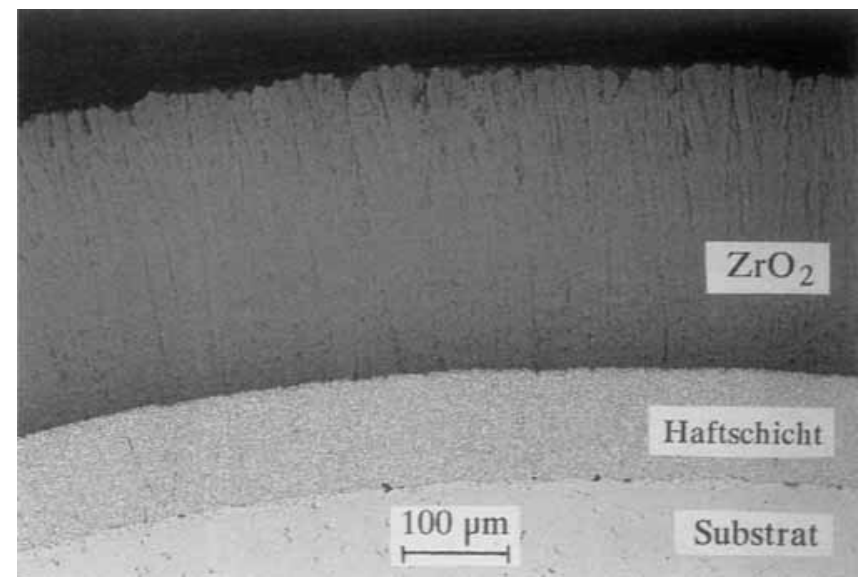

Abb. 3. Schliffbild einer elektronenstrahlgedampften Zirkonoxidschicht mit stengelförmiger Mikrostruktur

Fig. 3. Metallographic section of an electron beam vapor deposited zirconia coating with columnar microstructure
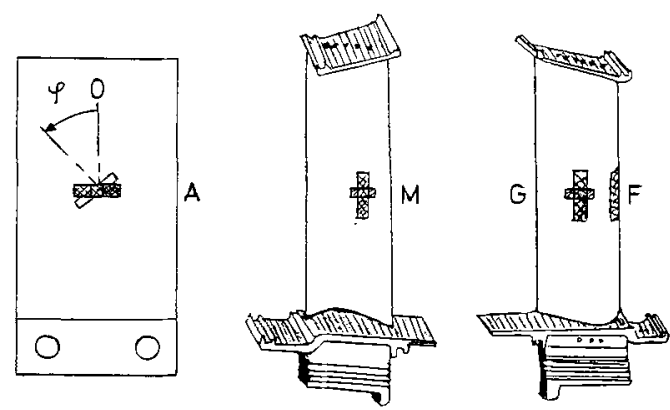

Abb. 4. Lage der Meßstellen und MeBrichtungen; links: Flachprobe, Mitte: konkave Oberfläche, rechts: konvexe Oberfläche

Fig. 4. Position of measuring spots and directions of stress measurements; left: flat specimen, middle: concave surface, right: convexe surface

Schaufeln sowie die Meßrichtung der Eigenspannungsmessungen sind in $A b b .4$ skizziert. Die Größe der bestrahlten Meßflächen betrug im allgemeinen etwa $12 \mathrm{~mm} \times 1,5 \mathrm{~mm}$. 
Lediglich bei Spannungsmessungen in Schaufellängsrichtung und bei Messungen an der Eintrittskante mußte wegen der Oberflächenwölbung eine kleinere Meßfläche von etwa $2 \mathrm{~mm} \times 1,5 \mathrm{~mm}$ gewählt werden (Positionen F, G, M).

Für die röntgenografische Spannungsmessung wurden die Linienlagen nach der Schwerpunktmethode bestimmt. Die Meßtiefe betrug bei dem (620)-Peak $(2 \vartheta=144,7$ Grad) je nach $\psi$-Winkel zwischen 3 bis $7 \mu \mathrm{m}$.

Wie in der vorangegangenen Untersuchung [2] an plasmagespritzten Schichten wurde die Auswertung mit einer röntgenografischen Elastizitätskonstanten $1 / 2 \mathrm{~s}_{2}=$ $26 \cdot 10^{-6} \mathrm{~mm}^{2} / \mathrm{N}$ vorgenommen. Dieser Wert wurde aus dem makroskopischen Elastizitätsmodul von $50 \mathrm{GPa}$ für plasmagespritzte Schichten [3,4] und einer Querkontraktionszahl $v=0,3$ errechnet.

Die Problematik dieser Vorgehensweise wurde bereits in der früheren Untersuchung diskutiert. Während für plasmagespritzte Schichten (Abb. 2) mit poröser Mikrostruktur die Wahl des für dieses Material sehr niedrigen EModuls gerechtfertigt erscheint, ist diese Vorgehensweise bei den elektronenstrahlgedampften Schichten fragwürdig. Bei diesen Schichten mit porenfreien Stengelkristallen (Abb. 3) erscheint auch die Wahl des mit 200 bis $250 \mathrm{GPa}$ sehr viel größeren E-Moduls für kompaktes Zirkonoxid plausibel. Solange jedoch die Werte für die röntgenographischen Elastizitätskonstanten von elektronenstrahlgedampften Schichten wegen fehlender E-Modul-Messungen noch unsicher sind, bietet der gewählte $1 / 2 \mathrm{~s}_{2}$-Wert den Vorteil, daß die Ergebnisse dieser Messungen hinsichtlich der Meßunsicherheiten unmittelbar mit jenen der vorangegangenen Untersuchungen verglichen werden können. Eine Umrechnung der Ergebnisse in Spannungswerte, denen eine andere röntgenographische Elastizitätskonstante ${ }^{1 / 2} s_{2}{ }^{\prime}$ zugrunde gelegt wird, ist durch Multiplikation der Wert mit dem Faktor $\mathrm{s}_{2} / \mathrm{s}_{2}{ }^{\prime}$ bzw. $\mathrm{E}^{\prime} / \mathrm{E}$ (bei gleichbleibendem $v$ ) zu bewerkstelligen. Im vorliegenden Fall könn-
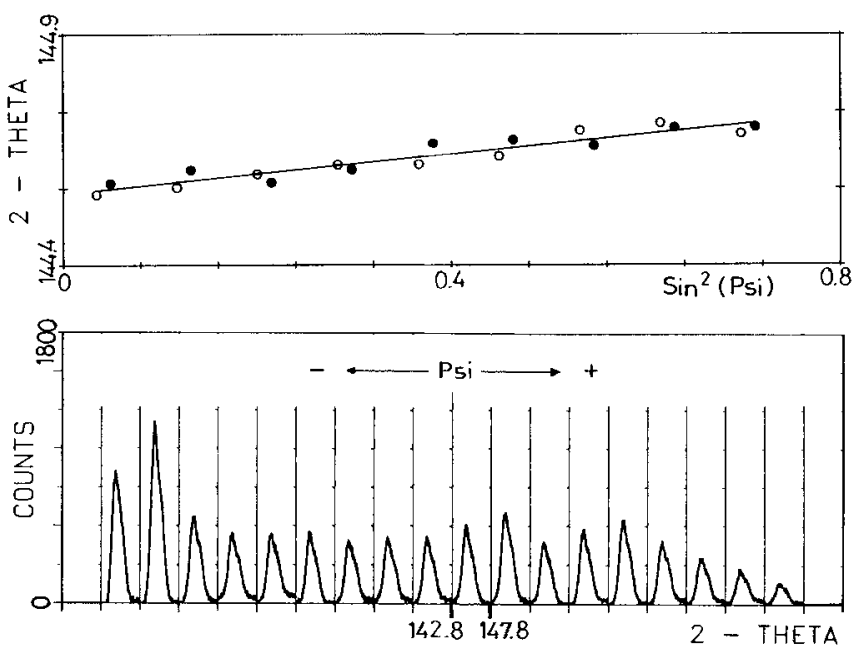

Abb. 5. Schaufel mit geringer Textur, Meßstelle G; Spannungsmessung in Schaufellängsrichtung $\left(\varphi=180^{\circ}\right)$; $\sin ^{2} \psi$-Verteilung, ( + bzw. 0 : positive; - bzw. $\bigcirc$ : negative $\psi$-Winkel); Eigenspannungswert: $-20 \mathrm{~N} / \mathrm{mm}^{2}$ (Druckeigenspannung), statistische Meßunsicherheit $\pm 2 \mathrm{~N} / \mathrm{mm}^{2}$

Fig. 5. Turbine blade with weak texture, measuring spot $\mathrm{G}$; stress determination in longitudinal direction $\left(\varphi=180^{\circ}\right), \sin ^{2} \psi$ distribution, positive $(+/ 0)$ and negative $(-/ O) \psi$-angles, stress value $-20 \mathrm{~N} / \mathrm{mm}^{2}$ (compressive stresses), statistical error \pm 2 $\mathrm{N} / \mathrm{mm}^{2}$
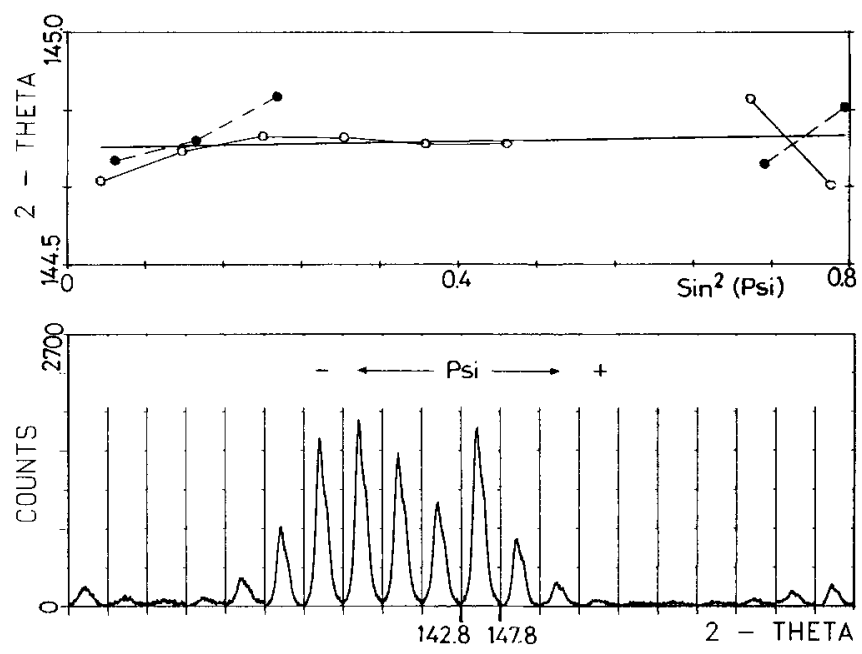

Abb. 6. Wie Abb. 5; Schaufel mit starker Textur, unzureichend auswertbare $\sin ^{2} \psi$-Verteilung

Fig. 6. As for fig. 5; turbine blade with strong texture, evaluation of $\sin ^{2} \psi$-distribution is difficult

ten sich dadurch bis um den Faktor 5 größere Eigenspannungswerte ergeben. Die aus den Ergebnissen zu ziehenden Folgerungen bleiben jedoch von dieser Betrachtungsweise unberührt. Aufgrund von Wiederholungsmessungen und statistischen Schwankungen der $\sin ^{2} \psi$-Verteilungen wurde die Meßunsicherheit der Eigenspannungswerte auf $\pm 5 \mathrm{~N} / \mathrm{mm}^{2}$ geschätzt. Dies gilt jedoch nur für gut auswertbare Messungen an Proben mit geringer Textur. Bei Proben mit stark ausgeprägter Textur lagen große statistische Schwankungen und nichtlineare $\sin ^{2} \psi$-Verteilungen vor. In den $A b b .5$ und 6 sind zwei typische $\sin ^{2} \psi$-Verteilungen dargestellt, die das Spektrum der Ergebnisse von ,gut auswertbar" bis „unzureichend auswertbar" charakterisieren.

\section{Ergebnisse der röntgendiffraktometrischen Messungen}

In den Diffraktogrammen waren ausschließlich Linien der tetragonalen Zirkonoxidphase vorhanden, $A b b .7 a$. Im Gegensatz zu plasmagespritzten Schichten [2], $A b b .7 b$, fehlte der (111)-Peak der monoklinen $\mathrm{ZrO}_{2^{-}}$ Phase und war nicht einmal andeutungsweise erkennbar. Es wurden auch keine Linienverbreiterungen nach thermischer Beanspruchung festgestellt.

Das Verhältnis der Linienintensitäten zwischen den Diffraktogrammen der aufgedampften Schicht und einer abgelösten, pulverisierten Schicht zeigt zum Teil starke Abweichungen, die auf das Vorhandensein von Texturen hinweisen. Diese waren jedoch sehr unterschiedlich stark ausgeprägt. Im Gegensatz dazu war bei den plasmagespritzten Schichten keine Textur erkennbar. Da eine quantitative Texturmessung mit der vorhandenen Meßeinrichtung nicht möglich war, wurden an einigen Proben qualitative Messungen an der für die röntgendiffraktometrische Spannungsmessungen verwendeten (620)-Linie vorgenommen.

Die $A b b .8$ und 9 zeigen Linienprofile bei einem $\psi$ Winkel von $57^{\circ}\left(\sin ^{2} \psi=0,7\right)$ unter verschiedenen 

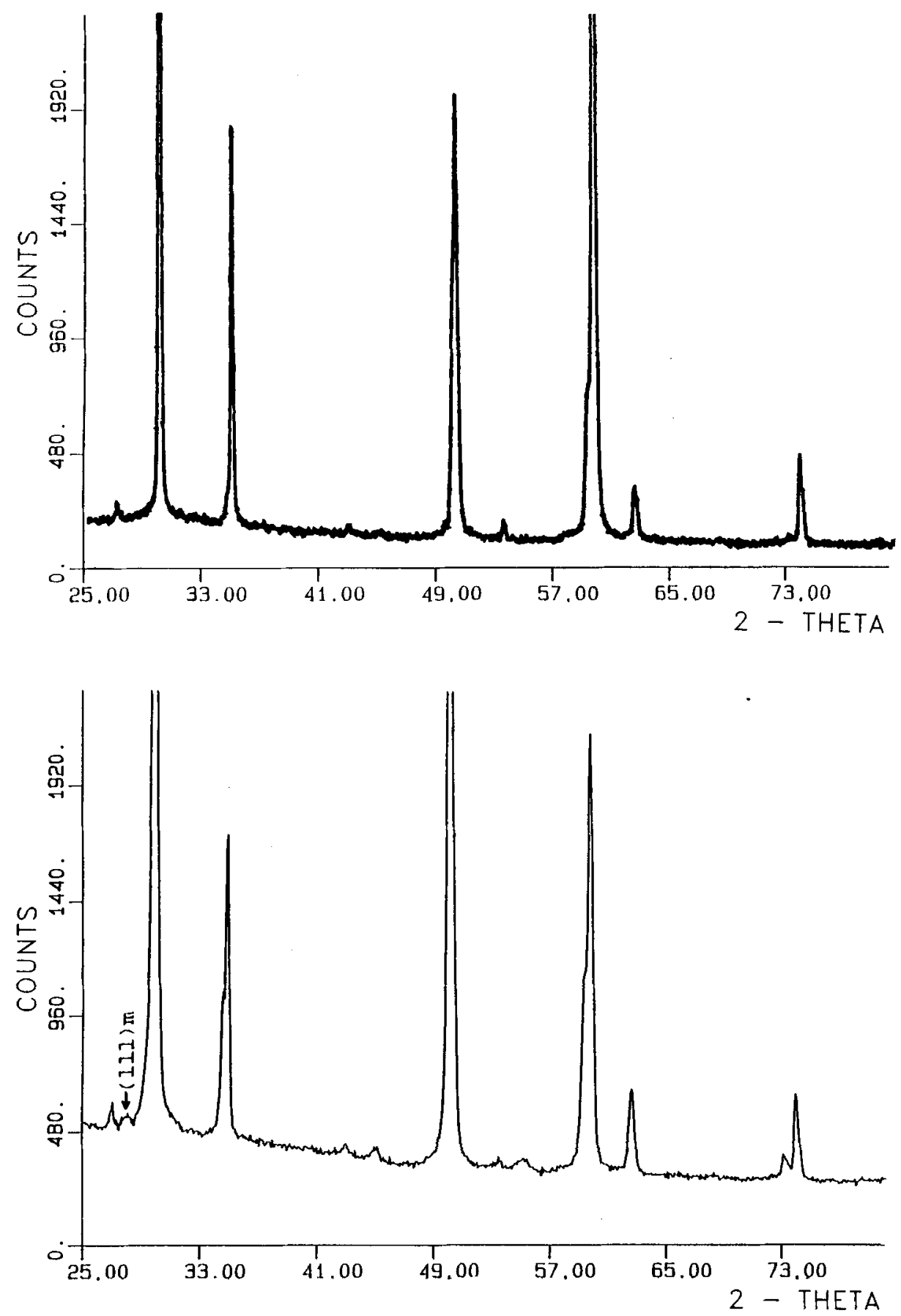

Abb. 7a. Diffraktogramm einer elektronenstrahlgedampften Schicht mit ausschließlich tetragonaler $\mathrm{ZrO}_{2}$-Phase Fig. 7. a. Diffraction pattern of an electron beam vapor deposited coating with only tetragonal $\mathrm{ZrO}_{2}$-phase
Meßrichtungen, d.h. $\varphi$-Winkeln (Abb. 4). In Abb. 8 unterscheiden sich die Intensitäten in Abhängigkeit vom $\varphi$-Winkel nur geringfügig. Die Textur ist offensichtlich nur relativ schwach ausgeprägt. Unter diesen Bedingungen erscheint eine Eigenspannungsmessung mit Auswertung nach dem $\sin ^{2} \psi$-Verfahren möglich.

Abb. 9 stellt den Fall einer sehr stark ausgeprägten Textur dar. Hier ist die Intensität der (620)-Linie bei den meisten $\varphi$-Winkeln so schwach, daß eine Linienlage nicht mehr mit einer für eine Spannungsmessung ausreichenden Genauigkeit bestimmbar ist. Die Auswertung nach dem $\sin ^{2} \psi$-Verfahren erscheint fraglich oder nur bedingt möglich. Auffallend war, daß der Texturzustand sich weniger zwischen einzelnen Meßstellen als vielmehr zwischen ein- zelnen Proben unterschied, d.h. die Charakterisierung „gut" und ,schlecht" auswertbar für die röntgenografischen Spannungsmessungen traf jeweils für alle Meßstellen einer Probe zu.

\section{Ergebnisse der röntgenografischen Eigenspannungsmessungen}

Eigenspannungsmessungen wurden an insgesamt 7 Flachproben und 11 zum Teil thermoermüdeten Schaufeln durchgeführt. An zwei Schaufeln wurden Messungen vor und nach der thermozyklischen Beanspruchung vorgenommen. 


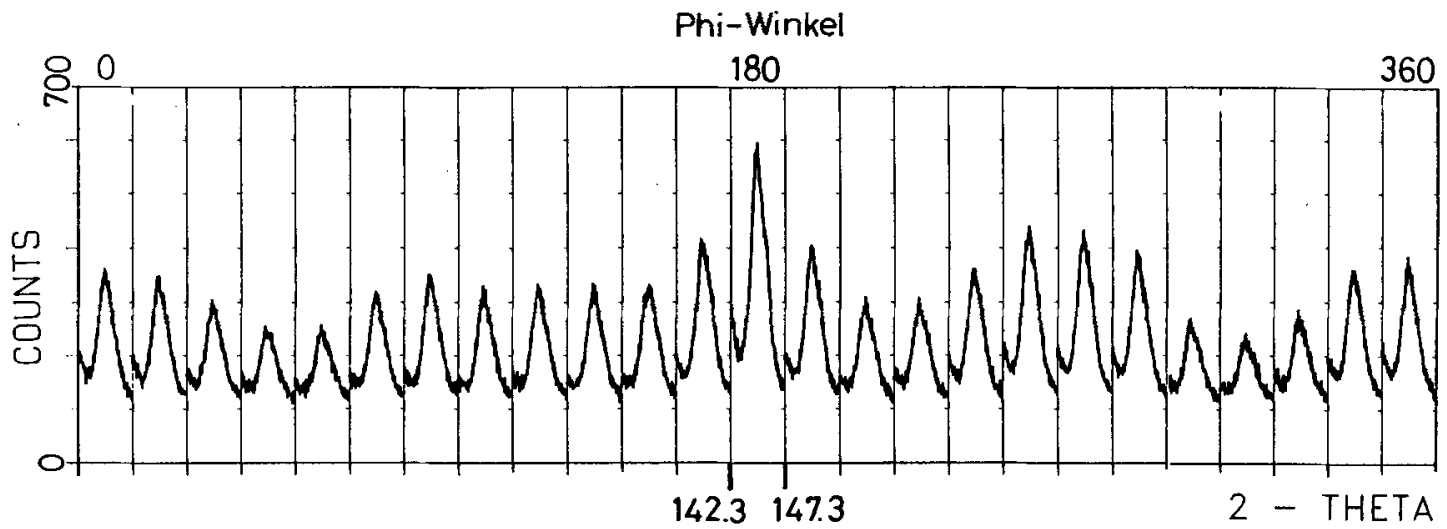

Abb. 8. Qualitative Texturmessung an einer Turbinenschaufel (Meßstelle G). (620)-Linie $\left(142,3^{\circ} \leq 2 \vartheta \leq 147,3^{\circ}\right), \psi 57^{\circ}\left(\sin ^{2} \psi=0,7\right)$, $\varphi=0^{\circ}$ bis $360^{\circ}$ in Abständen von jeweils $15^{\circ}$, geringe Textur

Fig. 8. Qualitative texture measurement on a turbine blade (measuring spot $G) ;(620)$-peak $\left(142,3^{\circ} \leq 2 \vartheta \leq 147,3^{\circ}\right), \psi=57^{\circ}\left(\sin ^{2} \psi=\right.$ $0,7), \varphi=0$ till $360^{\circ}$ with $15^{\circ}$ intervals, weak texture

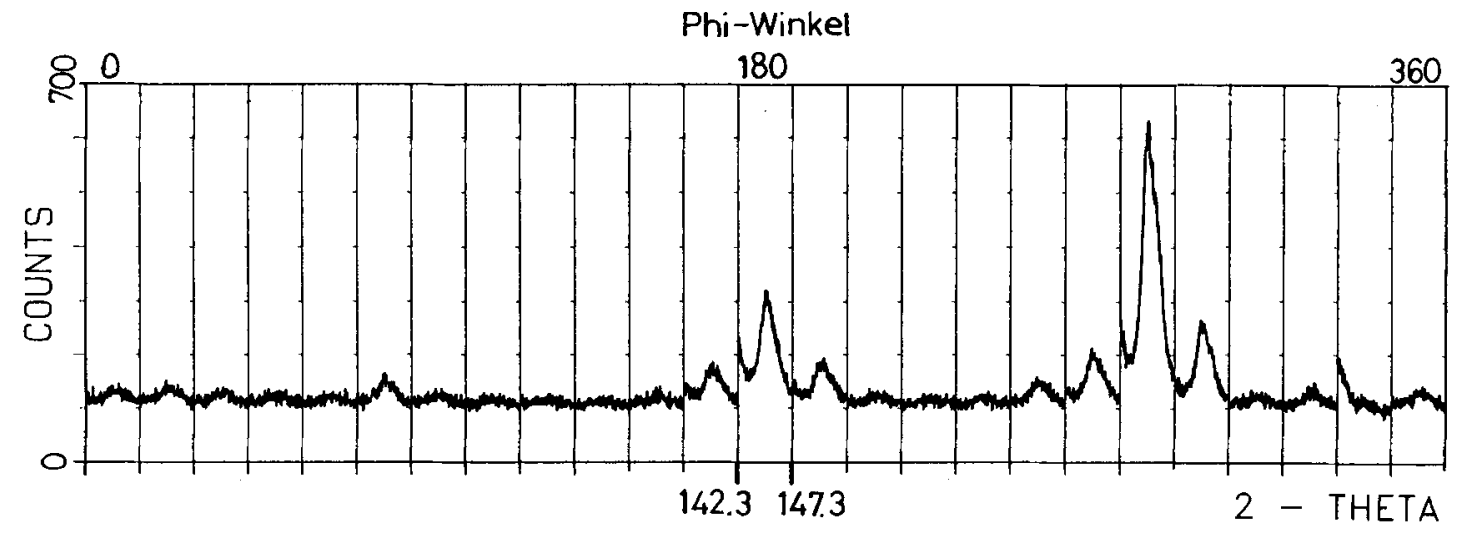

Abb. 9. Wie Abb. 8; Schaufel mit stark ausgeprägter Textur

Fig. 9. As for fig. 8, blade with strong texture

An allen Meßstellen wurden Druckeigenspannungen gemessen. Bei den gut auswertbaren Messungen lagen die Meßwerte zwischen -3 und $-37 \mathrm{~N} / \mathrm{mm}^{2}$ und bei den schlecht auswertbaren Messungen zwischen -3 und $-52 \mathrm{~N} / \mathrm{mm}^{2}$. Wegen der Vielzahl der teilweise doch stark streuenden Einzelwerte werden zur Charakterisierung der Eigenspannungen im folgenden nur Mittelwerte und Standardabweichungen angegeben.

\subsection{Richtungsabhängigkeit der Eigenspannungen}

An den Meßstellen A (Flachproben) sowie G und $M$ (Schaufeln) wurden Spannungsmessungen in verschiedenen $\varphi$-Richtungen durchgeführt, um die Abhängigkeit der Größe der Eigenspannungen von der Probengeometrie zu untersuchen. Diese Messungen wurden an allen Flachproben und an 6 ausgewählten Schaufeln vorgenommen.

Nachfolgend sind die Mittelwerte aus mehreren Messungen aufgeführt:

- Flachproben, Messungen gut auswertbar:

Probenlängsrichtung $-20( \pm 4) \mathrm{N} / \mathrm{mm}^{2}$

Probenquerrichtung $-13( \pm 4) \mathrm{N} / \mathrm{mm}^{2}$

Meßstelle A, 5 Flachproben.
- Flachproben, Messungen schlecht auswertbar:

Probenlängsrichtung $-46( \pm 6) \mathrm{N} / \mathrm{mm}^{2}$

Probenquerrichtung $-20( \pm 7) \mathrm{N} / \mathrm{mm}^{2}$

Meßstelle A, 2 Flachproben.

Ähnliche Verhältnisse bezüglich Probenrichtung und GröBe der Eigenspannungen lagen auch bei den Schaufeln vor.

- Schaufeln, Messungen gut auswertbar:

Schaufellängsrichtung $-21( \pm 4) \mathrm{N} / \mathrm{mm}^{2}$

Schaufelquerrichtung $-13( \pm 4) \mathrm{N} / \mathrm{mm}^{2}$

Meßstelle G (Konvexseite), 2 Schaufeln, jeweils 6 Messungen.

- Schaufeln, Messungen schlecht auswertbar:

Schaufellängsrichtung $-41( \pm 10) \mathrm{N} / \mathrm{mm}^{2}$

Schaufelquerrichtung $-27( \pm .5) \mathrm{N} / \mathrm{mm}^{2}$

Meßstelle G (Konvexseite), 4 Schaufeln, jeweils 6 Messungen. 
- Schaufeln, Messungen gut auswertbar

Schaufellängsrichtung $-31( \pm 4) \mathrm{N} / \mathrm{mm}^{2}$

Schaufelquerrichtung $-22( \pm 7) \mathrm{N} / \mathrm{mm}^{2}$

Meßstelle M (Konkavseite), 2 Schaufeln, jeweils 6 Messungen.

Es scheint möglich, daß zwischen den Schichten der Flachproben und der Schaufeln mit gut und schlecht auswertbaren Messungen keine echten Eigenspannungsunterschiede vorhanden sind, sondern daß ein texturbedingter Meßeffekt vorliegt. Der Unterschied der Eigenspannungswerte in Längs- und Querrichtung scheint dagegen nicht mit Textureffekten oder unterschiedlicher Probengeometrie (Oberflächenk rümmung) in Verbindung zu stehen, da dieser Unterschied sowohl bei den Flachproben als auch bei den Schaufeln etwa gleich groß ist. Auch auf der konkaven Seite der Schaufeln liegen unterschiedliche Eigenspannungen in Längs- und Querrichtung vor.

Möglicherweise sind diese Eigenspannungsunterschiede zwischen Längs- und Querrichtung bei den Flachproben und Schaufeln (beidseitig) herstellungsbedingt und korrelieren mit der Orientierung der Proben in der Beschichtungsapparatur. Nicht völlig auszuschließen ist allerdings, daß grundsätzlich auch eine schwach ausgeprägte Textur eine Rolle spielt.

\subsection{Eigenspannungsänderung durch thermozyklische Beanspruchung}

Der Einfluß von thermozyklischer Beanspruchung auf den Eigenspannungszustand wurde an sechs Schaufeln gemessen. Am aussagekräftigsten waren Messungen, die an zwei Schaufeln an jeweils gleichen Meßstellen ( $G$ und $\mathrm{M})$ vor und nach thermozyklischer Beanspruchung vorgenommen wurden. Der Mittelwert von an korrespondierenden Meßstellen und -Richtungen gemessenen Differenzen der Eigenspannungen betrug $1( \pm 2) \mathrm{N} / \mathrm{mm}^{2}$ (11 Wertepaare). Außerdem wurden Messungen an vier weiteren Schaufeln durchgeführt, jeweils zwei mit und ohne thermozyklischer Beanspruchung. Diese Schaufeln fielen unter die Kategorie ,schlecht auswertbar". Bei diesen Schaufeln betrug die Differenz der Meßwerte $0( \pm 13) \mathrm{N} / \mathrm{mm}^{2}$ (Meßstelle G, 12 Wertepaare). Anhand dieser Ergebnisse erscheint eine Eigenspannungsänderung als Folge der thermozyklischen Beanspruchung unwahrscheinlich. Dabei ist allerdings zu berücksichtigen, daß die Meßstellen $G$ und $M$ nicht die am stärksten thermisch beanspruchten Schaufelbereiche sind. Diese liegen vielmehr an den Einund Austrittskanten (z. B. MeBstelle F -- Schaufeleintrittskante) und nur dort treten erfahrungsgemäß Beschädigungen in Form von feinen Rissen und Abplatzungen in der Wärmedämmschicht auf. Messungen an den Schaufelkanten sind aber relativ schwierig, erstens wegen der starken Oberflächenkrümmung und zweitens, weil die Einflüsse von Rissen und/oder Textur von irgendwelchen echten Eigenspannungsänderungen schwer zu trennen sind. Im Rahmen dieser Arbeit konnten nur vereinzelte Messungen an den Kanten thermozyklisch beanspruchter Schaufeln durchgeführt werden. Es war daher nicht möglich, aus den Meßergebnissen eine eindeutige Aussage abzuleiten.

\section{Diskussion}

Im Gegensatz zu plasmagespritzten Wärmedämmschichten aus Zirkonoxid konnten an elektronenstrahlgedampften Schichten keine signifikanten Unterschiede des Eigenspannungszustandes vor und nach thermischer Wechselbeanspruchung gemessen werden. Die Ursache hierfür dürfte mit der unterschiedlichen Mikrostruktur der Schichten in Verbindung stehen. Plasmagespritzte Schichten weisen eine poröse schichtartig aufgebaute Struktur auf, elektronenstrahlgedampfte Schichten dagegen eine Stengelstruktur (Abb. 2 und 3). Offenbar vermag der Verbund MetallKeramik mit stengelförmiger Zirkonoxidschicht aufgrund der in Normalenrichtung verlaufenden Grenzen zwischen den Stengelkristallen Verformungen infolge ungleichmäßiger thermischer Ausdehnungen von Schicht und Substrat besser, d. h. mit weniger irreversiblen Mikroverformungen zu ertragen als der Verbund mit plasmagespritzten Schichten, bei denen diese Mikroverformungen $z u$ relativ gut meßbaren Eigenspannungsänderungen in Richtung abnehmender Zug- bzw, zunehmender Druckeigenspannungen führte.

Bemerkenswert ist, daß die längeren Standzeiten bei den elektronenstrahlgedampften Schichten trotz Vorliegens von ähnlich hohen oder sogar höheren Druckeigenspannungen als bei den plasmagespritzten Schichten erhalten werden. In der trüheren Untersuchung [2] an plasmagespritzten Schichten waren ja Druckeigenspannungen in der Wärmedämmschicht und deren Anwachsen für das Abplatzen an konvex gewölbten Oberflächen verantwortlich gemacht worden. Da das Fehlen von Eigenspannungsänderungen nach thermozyklischer Beanspruchung der elektronenstrahlgedampften Schichten keinen unmittelbaren Rückschluß auf einen Versagensmechanismus zuläßt, erhebt sich die Frage, ob auch andere Prozesse als die Zunahme von Druckeigenspannungen die Rißbildung und Schichtabplatzungen im Schaufelkantenbereich begünstigen oder sogar maßgeblich verursachen.

Meier et al. [5] haben das Verformungsverhalten von plasmagespritzten und elektronenstrahlgedampften Schichten bei $540^{\circ} \mathrm{C}$ und Druckbeanspruchung gemessen. Sie ermittelten, daß die bei gleicher Dehnungsbeanspruchung auftretenden inelastischen Verformungen bei den plasmagespritzten Schichten um den Faktor 10 größer sind als bei den elektronenstrahlgedampften Schichten und daß derartig hohe Verformungen bei den elektronenstrahlgedampften Schichten erst bei deutlich höheren Spannungen auftreten. Dies erklärt das Fehlen von Eigenspannungsänderungen und auch die im allgemeinen deutlich längeren Standzeiten der elektronenstrahlgedampften Schichten gegenüber plasmagespritzten Schichten.

Miller [6] führt das Abplatzen von plasma- und elektronenstrahlgedampften Schichten in erster Linie auf eine fortschreitende Oxidation der Haftschicht zurück. Er schließt dies aus thermozyklischen Versuchen mit Temperaturgradienten bis $1100^{\circ} \mathrm{C}$ mit großen Unterschieden der Standzeiten in oxidierender oder inerter Atmosphäre (Argon). Seine Rechnungen mit Finiten Elementen bei einer als sinusförmig angenommenen Grenzfläche zwischen Haftschicht und Keramik ergaben bei Oxidbildung Hinweise auf Eigenspannungsänderungen in Normalenrichtung, die ein Abplatzen begünstigen.

Meier et al. [5] haben speziell elektronenstrahlgedampfte Schichten unter der Hypothese des Schichtversagens durch Haftschichtoxidation untersucht und auf dieser Basis ein 
Versagensmodell entwickelt. Auch sie führen das Schichtversagen auf eine zunehmende Oxidbildung an der Haftschicht zurück, machen dafür aber den Aufbau von Spannungen in der Schichtebene verantwortlich. Diese Spannungen resultieren aus Fehlpassungen zwischen der Wärmedämmschicht und den Oxiden der Haftschicht. Als Folge dieser Fehlpassung treten beim thermischen Zyklen nichtelastische Verformungen auf, die zu einem Versagen an der Oxidschicht führen. Das Versagensmodell von Meier et al., das an zylinderförmigen Proben in thermischen Beanspruchungszyklen mit unterschiedlichen Zyklenspektren überprüft wurde, lieferte Werte für Lebensdauern der Schichten, die von den gemessenen Lebensdauern um max. $20 \%$ abwichen.

Für ein frühzeitiges Schichtversagen wie bei den früher untersuchten plasmagespritzten Schichten mit Druckeigenspannungen kann die Bildung von Haftschichtoxiden nicht maßgeblich sein, da dieser Vorgang relativ lange Beanspruchungszeiten bei hohen Temperaturen in oxidierender Atmosphäre erfordert. Bezüglich des langfristigen Schichtversagens erscheint dieses Modell aber sehr plausibel. Letzten Endes basiert das Schichtversagen aber auch bei diesem Modell auf der Änderung von Spannungszuständen, die meßbar sein sollten.

Da diese Spannungsänderungen an der Grenzfläche zum Substrat auftreten, sind ihre Auswirkungen an der Oberfläche der Wärmedämmschichten, an der röntgenografisch die Eigenspannungen gemessen werden, möglicherweise aber so klein, daß sie im Bereich der Meßunsicherheit liegen. Hinzu kommt der Umstand, daß im Bereich der Meßstellen $M$ und $G$ die Schichten thermisch weniger beansprucht werden als an den Schaufelkanten, wo die Schichten am Ende ihrer Lebensdauer abplatzen. An den Meßstellen $M$ und $G$ sind daher deutlich kleinere Eigenspannungsänderungen aufgrund von Haftschichtoxidation zu erwarten, als im Bereich der Schaufelkante. Aufschluß darüber, ob die Bildung von Haftschichtoxiden zu meßbaren Eigenspannungsänderungen führt, können daher nur weitere Messungen im Bereich der Schaufelkanten (Meßstelle F) oder an Rundproben erbringen, bei denen Eigenspannungen leichter gemessen werden können.

Bei den früheren Versuchen mit plasmagespritzten Schichten hatten sich Zugeigenspannungen als positiv für die Schichtlebensdauer erwiesen. Sogar Risse in den Wärmedämmschichten bei Kriechversuchen führten nicht zum Abplatzen der Schichten. Nimmt man an, daß ein Schichtabplatzen an Haftschichtoxiden ebenfalls auf Druckspannungen in der Schichtebene und die daraus resultierende Normalenkomponente bei konvexer Oberflächenkrümmung zurückzuführen ist, so sollten sich Zugeigenspannungen in der Wärmedämmschicht auch hier positiv auswirken. Allerdings wird eine ähnlich hohe Steigerung der Lebensdauer wie bei den plasmagespritzten Schichten nicht zu erwarten sein.

Brindley und Miller [7] berichten über einen weiteren Versagensmechanismus, welcher zur Folge hat, daß bei elektronenstrahlgedampften Schichten die Tendenz zum Abplatzen von kleineren Schichtbereichen besteht, als dies bei plasmagespritzten Schichten der Fall ist. Sie führen diesen Versagensmechanismus bei den elektronenstrahlgedampften Schichten auf das Zusammensintern von einzelnen Stengeln zurück. Da aus diesem Versagensmodell auf örtlich eng begrenzte Eigenspannungsänderungen geschlossen werden muß, steht es mit den vorgefundenen Meßergebnissen an den Meßstellen $M$ und $G$ nicht im
Widerspruch. Bei den vorgenommenen Eigenspannungsmessungen dürfte die Meßfläche im Verhältnis zu den Sinterbereichen immer noch relativ groß sein, so daß Eigenspannungsunterschiede zwischen möglichen Sinterbereichen und Umgebung entsprechend den Flächenanteilen nicht mit ausreichender Signifikanz nachweisbar sind. Weitere Eigenspannungsmessungen in Verbindung mit einer gezielten mikroskopischen Suche nach Sinterbereichen könnten hier Klarheit verschaffen.

Bei den thermisch wechselbelasteten Turbinenschaufeln fällt auf, daß die beiden Schaufeln mit starker Textur (Eigenspannungsmessungen ,schlecht auswertbar") nur eine relativ geringe Anzahl von 500 bis 1300 thermische Zyklen bis zum Auftreten von ausgeprägten Abplatzungen an den Ein- und Austrittskanten erreichten. Die zwei anderen, ebenfalls thermoermüdeten Schaufeln besaßen nur relativ schwach ausgeprägte Texturen und zeigten nach 2000 Thermozyklen Schädigungen jeweils nur in Form eines Risses an Ein- bzw. Austrittskante. Möglicherweise besteht ein Zusammenhang zwischen der Stärke der Textur und dem Zeitpunkt des Auftretens von Abplatzungen bei Thermoermüdung. Entsprechende Messungen an Schichten mit vergleichsweise kurzer und langer Lebensdauer könnten diese Frage klären.

Von Interesse wäre auch, ob und in welcher Art und Weise die Herstellungsbedingungen Einflu $\beta$ auf die Textur nehmen. Der große Unterschied der Texturausprägung zwischen den "gut" und „schlecht" auswertbaren Proben und der geringe Unterschied der Texturausprägung innerhalb einer Probe lassen auf unterschiedliche Herstellungsbedingungen schließen. Auch die Eigenspannungsunterschiede in Probenlängs- und -querrichtung weisen auf besondere herstellungsbedingte Einflüsse bezüglich der Schichtausbildung hin. Vermutlich besteht ein Zusammenhang zwischen der Größe der Eigenspannungen und der Orientierung der Proben in der Beschichtungsanlage.

\section{Danksagung}

Die Autoren danken dem Bundesministerium der Verteidigung für die Bereitstellung von Mitteln. Für die gewissenhafte Durchführung der röntgendiffraktometrischen Messungen wird Herrn F. Wachinger besonders gedankt.

\section{Literatur}

1. S. Schneiderbanger, W. Wei, MTU München GmbH; unveröffentlichte Ergebnisse.

2. H. Richter, T. Cosack, S. Schneiderbanger, „Röntgenografische Eigenspannungsmessungen an plasmagespritzten Wärmedämmschichten aus Zirkonoxid vor und nach thermischer und mechanischer Beanspruchung; Mat.-wiss. u. Werkstofftechnik, 24 (1993) 183-189.

3. W. J. Lackey et al., Ceramic Coating for Advanced Heat Engines-A Review and Projection; Advanced Ceramic Materials Vol. 2 Nr. 1 (1987) 24-30.

4. P. Bock et al., Plasma Sprayed Zirconia Coatings: Advances in Ceramics, Vol, 12 Am. Cer. Soc. Columbus OHIO USA (1984) $488-502$.

5. S. M. Meier, D. M. Nissley, K. D. Sheffler, T. A. Cruse, "Thermal Barrier Coating Life Prediction Model Development", ASME Paper Nr. 91-GT-40. 
6. R. A. Miller, "Current Status of Thermal Barrier Coatings- An Overview", Surface Coatings and Technology 30 (1987) 1-11.

7. W. J. Brindley, R. A. Miller, Thermal Barrier Coating Evaluation Needs; Conference on Nondestructive Evaluation of Modern Ceramics Columbus, 9. 7.-12. 7. 1990, American Society for Nondestructive Testing, Columbus OHIO USA (1990) S. 70-76.
Anschriften der Autoren: Dr. Hans Richter, Wehrwissenschaftliches Institut für Materialuntersuchungen (WIM), Landshuter Str. 70, 85435 Erding; Prof. Dr. William Wei, Universiteit Twente, Fakulteit Werktuigbouw, Postbus 217, NL-7500 AE Enschede, Stefan Schneiderbanger, MTU-Deutsche Aerospace, Dachauer Str. 665, 80995 München.

[T 737]

\section{Neue Textilien durch Anwendung der Plasmatechnik}

\section{Das Bundesministerium für Bildung, Wissenschaft, Forschung und Technologie (BMBF) teilt mit:}

Unser Land benötigt vorausschauende Konzepte sowie Fortschritte bei den technologischen Grundlagen und beim Transfer von technisch-wissenschaftlichen Ergebnissen, um den Herausforderungen der Zukunft gerecht zu werden. Wissenschaft, Wirtschaft und Politik sind in besonderer Weise aufgefordert, ihren Beitrag hierzu zuleisten. Es geht insbesondere um zukunftssicheres Wirtschaftswachstum, um Arbeitsplätze, stabile Einkommen, soziale Sicherheit und einen hohen Umweltstandard.

Ein Instrument zur besseren Zukunftsgestaltung ist der Dialog zwischen den beteiligten Einrichtungen von Wissenschaft, Wirtschaft und Staat. Das Bundesministerium für Bildung, Wissenschaft, Forschung und Technologie (BMBF) stellt sich diesen Anforderungen und bekennt sich zu seiner Rolle als Moderator in diesem Dialogprozeß.

Im Rahmen dieses Dialoges hat das VDI-Technologiezentrum, Projektträger des BMBF für den Förderbereich „Physikalische Technologien", in Zusammenarbeit mit dem Gesamtverband der Textilindustrie in der Bundesrepublik Deutschland - Gesamttextil e.V. in Düsseldorf ein Fachgespräch mit 40 Experten aus Wissenschaft und Industrie zum Thema „Plasmaanwendungen in der Textilindustrie"veranstaltet. Ziel der Tagung war es, wichtige neue Entwicklungen auf dem Gebiet der Plasmaanwendungen in der Textil- industrie zu identifizieren und hinsichtlich ihres Potentials zu bewerten. Außerdem sollten neue Ergebnisse auf dem Gebiet der Plasmatechnik im Hinblick auf Anwendungen in der Textilindustrie dargestellt und bewertet werden.

Technische Textilien gewinnen für die Zukunft der deutschen Textilindustrie, die zu rund 90 Prozent aus mittelständischen Unternehmen mit rund 160000 Beschäftigten besteht, eine immer gröBere Bedeutung. Hierzu zählt ein wachsendes Anwendungsspektrum in den Bereichen Schutzbekleidungen, Verkehrs- und Bauwesen, Umweltschutz sowie in zahlreichen technischen Einsatzgebieten. Bedingt durch diese enorme Anwendungsbreite textiler Produkte ergeben sich spezifische Anforderungen an die Textilien, die sich nur durch den Einsatz innovativer Bearbeitungsverfahren erzielen lassen. Neben der Zerreißfestigkeit und der Elastizität der Gewebe gehören zu diesen Eigenschaften Z. B. auch wasserabweisende und säurebetsändige Oberflächen sowie eine hohe Temperturbeständigkeit. Die Plasmatechnologie bietet das notwendige Potential zur Erzielung der gewünschten Eigenschaften und damit zur Hertsellung völlig neuartiger Gewebe. Wenn wir auf dem weltmarkt konkurrenzfähig bleiben wollen, müssen neue physikalische Verfahren und Hochtechnologien zur Oberflächenmodifizierung von Fasern rasch definiert und zügig in Anwendungen umgesetzt werden.

Die Ergebnisse des Fachgesprächs soll deshalb in einen strategischen Dialog zur Plasmatechnologie einfließen, zu dem das BMBF im Herbst Vertreter von Industrieverbänden, Unternehmen sowie Wissenschaftler und Forschungsinstitute einladen wird. 